\title{
The effect of the stress far field on the crack tip behaviour
}

\author{
A. Piva
}

Received on December 5th, 1978

\section{ABstract}

The boundary value problem of an infinite purely elastic sheet with a traction-free crack loaded with a uniform shear and biaxial tension at infinity is solved. It is shown that the singular terms of stress and displacement functions are inadequate to predict the direction of initial crack extension. The independence of the $J$ vector of the biaxial parameter is also proved.

\section{RIASSUNTO}

Risolvendo il problema al contorno di una lastra piana, sottoposta all'infinito ad un campo di sforzo biassiale e di scorrimento, con un " crack" libero da trazioni, si mette in evidenza il contributo dei termini non singolari sul campo degli sforzi e dello spostamento al "tip» del crack e l'influenza che tali termini hanno sui criteri locali di diramazione. Si dimostra inoltre l'indipendenza del vettore $J$ dai termini non singolari.

\footnotetext{
* Istituto di Fisica -Università di Bologna.
} 


\section{1 - INTRODUCTION}

The knowledge of stresses and displacements near a fault is one of the main purposes of Geophysics. However, for a fault model which allows a mathematical description we must concern ourselves with fairly idealized situations for the present. In this paper we shall start with the two-dimensional model of a crack which has received much attention since the work of Inglis (1913) and has been the main source of progress in fracture mechanics. As far as the crack tip behaviour is concerned some other problems can be of geophysical interest as the real effect of the far field on the crack tip stress and displacement distributions and on the crack propagation criteria.

In recent publications (Carpinteri, 1968; di Tommaso ed al., 1976; Eftis, 1977; Viola, 1978) it has been shown that the singular for a plane cracked body subjected to biaxial tension at infinity are insufficient for a correct description of the crack tip behaviour.

The arbitrary omission of non singular terms depending on the biaxial load parameter $K$ causes, in general, incorrect predictions on physical quantities such as the maximum tensile stress, the elastic strain energy density and the local elastic strain energy rate.

It follows that the crack propagation criteria such as the maximum tensile stress criterion and the minimum strain energy density criterion will fail in their predictions (Carpinteri, 1968; Di Tommaso et al., 1976; Eftis, 1977; Viola, 1978). Apparently surprising is the independence of the biaxial load parameter, shown in (Eftis, 1977) of the $J_{r}$-integral which is connected with a crack extension criterion, along the direction of the crack, through the attainment of a critical value $J_{1 c}$. This independence was justified by Eftis (1977) considering that the $J_{l}$-integral is connected with the global strain energy rate which appears to be independent of the horizontal load applied parallel to the plane of the crack. It seems then that the biaxial load affects only the local quantities. 
The aims of this paper are to describe the elastic fields of stresses and displacements near the tip of a central crack in an infinite elastic sheet loaded with a uniform shear and biaxial tension at infinity. Some considerations are made on the influence of non singular terms on crack propagation criteria the independence of the $J$ vector from the biaxial load parameter is proved.

2. - STATEMENT AND SOLUTION OF THE ELASTIC PROBLEM

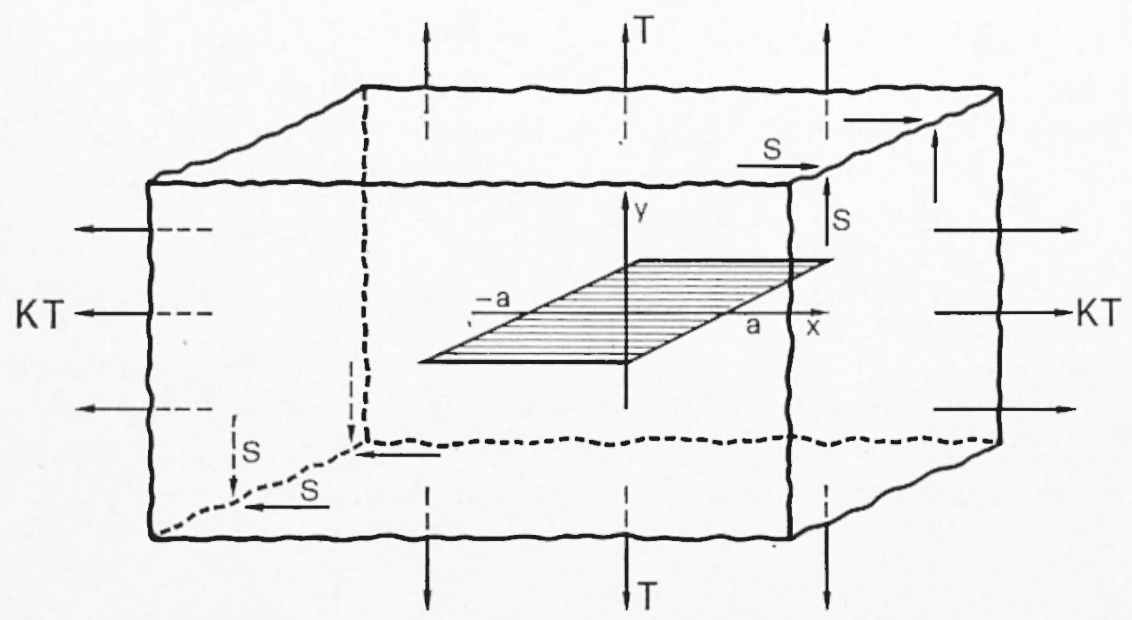

Fig. 1

We consider an infinite purely elastic body with a plane crack loaded at infinity, as shown in fig. 1. The solution of the elastic problem under plane strain or plane stress conditions is reduced to the evolution of the potential functions $\Omega(z)$ and 
$\theta(z)$ holomorphic in a plane parallel to the $x y$ plane and cut along the trace of the crack. These functions are related to stresses and displacement functions by the well-known relations [7] (Muskelishvili et al., 1953)

$$
\begin{aligned}
& \sigma_{x x}+\sigma_{y y}=z\left[\Omega^{\prime}(z)+\Omega^{\prime}(\bar{z})\right] \quad, z=x+i y \\
& \sigma_{y y}-i \sigma_{x x}=\Omega^{\prime}(z)+\Omega^{\prime}(\bar{z})+(z-\bar{z}) \bar{\Omega}^{\prime \prime}(z)-\vartheta^{\prime}(\bar{z}) \\
& Z \Vdash D=x \Omega(z)-\Omega(\bar{z})+(\bar{z}-z) \bar{\Omega}^{\prime}(\bar{z})+\vartheta(\bar{z})
\end{aligned}
$$

$$
x=\left\{\begin{array}{cc}
3-4 \vee & \text { for plan strain } \\
3-\vee & \text { for plane stress } \\
1+\vee &
\end{array}\right.
$$

where $D=u+i v$ is the complex displacement, $\mu$ is the shear modulus and $v$ is the Poisson ratio. We have for the stress distribution the following far field conditions:

$$
\sigma_{x x}=K T \quad, \sigma_{y y}=T \quad, \sigma_{x y}=S \quad ;|z| \rightarrow \infty
$$

where $K$ is a real constant which gives a measurement of the biaxiality of the stress field at infinity. Moreover, we have the boundary conditions on the traction-free crack surface:

$$
\sigma_{y y}-i \sigma_{x y}=0 \quad, \quad y=0 \pm \quad, \quad|x|<\alpha
$$


from [2.1], [2.2] and assuming zero rotation at infinity we obtain for the following behavior for the derivatives of the potential functions:

$$
\begin{aligned}
& \Omega^{\prime}(z)=\frac{(K-1) I+2 i S}{4}+0\left(\frac{1}{|z|^{2}}\right) \\
& \vartheta^{\prime}(z)=\frac{(K-1) I+2 i S}{2}+0\left(\frac{1}{|z|^{2}}\right)
\end{aligned}
$$

Moreover, after substitution of [2.1 $]_{2}$ into [2.3] we obtain the Hilbert problem:

$$
\begin{gathered}
{\left[2 \Omega^{\prime}(x)-\vartheta^{\prime}(x)\right]^{+}+\left[2 \Omega^{\prime}(x)-\vartheta^{\prime}(x)\right]^{-}=0 \quad,|x|<x} \\
\vartheta^{\prime}(x)-\vartheta^{\prime}(x)=0 \quad,|x| \leq x
\end{gathered}
$$

Making use of [2.4] we obtain the solution of [2.5] in the following form:

$$
\begin{gathered}
\Omega^{\prime}(z)=\frac{(K \cdot-1) T+2 i S}{4}+\frac{(I-i S)}{2} \frac{z}{X(z)} \\
\vartheta^{\prime}(z)=\frac{(K-1) T+2 i S}{2}
\end{gathered}
$$


where $X(z)=\left(z^{2}-a^{2}\right)^{1 / 2}$ is the Plemelej function of the problem (Muskelishvili, 1953), whose branch is selected in such a way that $X(z) \rightarrow z$ for large $|z|$. By integration of [2.6] we have also:

$$
\begin{gathered}
\Omega(z)=\frac{(K-1) T+2 i S}{4} z+\frac{T-i S}{2} X(z) \\
\vartheta(z)=\frac{(K-1) T+2 i S}{2} z
\end{gathered}
$$

By means of [2.1], [2.6] and [2.7] it is possible to derive the distributions of stresses and displacement functions in the plane.

3. - THE LOCAL CRACK TIP STRESS AND DISPLACEMENT COMPONENTS

Confining our attention to the crack tip region $0<|\zeta|=$ $=r \ll 1$, where $\zeta=z-a=r e^{i \varphi}$ (fig. 2), we can consider the following series of expansions:

$$
X(z)=a \cdot \sqrt{2}\left(\frac{\zeta}{a}\right)^{1 / 2}\left[1+\frac{1}{4} \frac{\zeta}{a}-\frac{1}{32}\left(\frac{\zeta}{a}\right)^{2}+\ldots\right]
$$

$$
\frac{z}{X(z)}=\frac{1}{\sqrt{2}}\left[\left(\frac{\zeta}{a}\right)^{1 / 2}+\frac{3}{4}\left(\frac{\zeta}{a}\right)^{1 / 2}-\frac{5}{32}\left(\frac{\zeta}{a}\right)^{3 / 2}+\ldots\right]
$$


To emphasize the singular behaviour of stresses near the tip we neglect all terms of order $(\zeta / a)^{1 / 2}$ and above after substitution of $[3.2)$ into $[2.6]_{1},[2.1]_{1}$ and $[2.1]_{2}$.

Moreover, in order to maintain the same approximation for the displacement we neglect terms of order $(\zeta / a)^{3 / 2}$ and above after substitution of [3.1] in $[2.7]_{\mathrm{I}}$ and $[2.1]_{3}$.

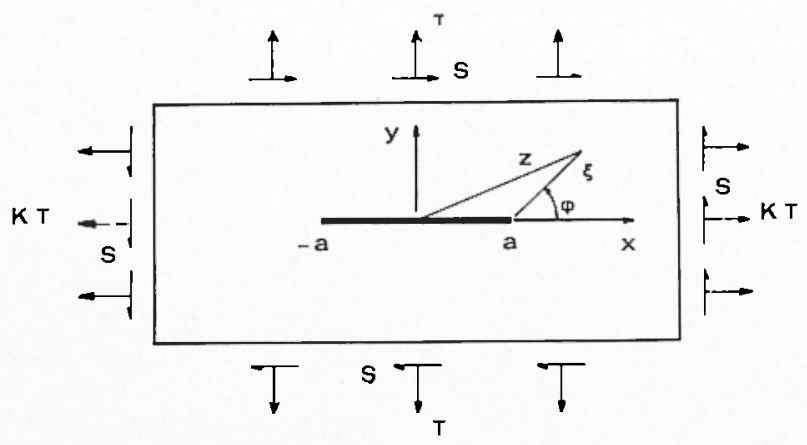

FIg. 2

For the fundamental stress combinations we obtain the following expressions:

$$
\begin{gathered}
\sigma_{x x}+\sigma_{y, \ldots}=A+\frac{\bar{S}_{1}}{\sqrt{\bar{\zeta}}}+\frac{S_{1}}{\sqrt{\bar{\zeta}}} \\
\sigma_{x x}-\sigma_{y y}+2 i \sigma_{x y}=A+\frac{S_{1} \zeta}{2 \bar{\zeta} \sqrt{-\bar{\zeta}}}-\frac{\bar{S}_{2}}{2 \sqrt{\bar{\zeta}}}
\end{gathered}
$$

with :

$$
A=(K-1) T, \quad S_{1}=\frac{K_{1}+i K_{2}}{\sqrt{2 \pi}} \quad, \quad S_{2}=\frac{K_{1}+3 i K_{2}}{\sqrt{2 \pi}}
$$


A. PIVA

where $K_{1}=T \sqrt{\pi a}$ and $K_{2}=S \sqrt{\pi a}$ are the stress intensity factors for the opening and sliding mode respectively. For the complex displacement function we obtain:

where:

$D=B+C \zeta+\frac{1}{4 \mu}\left[A \bar{\zeta}+2 x \bar{S}_{1} \sqrt{\bar{\zeta}}-\bar{S}_{2} \sqrt{\bar{\zeta}}-\frac{S_{1} \zeta}{\sqrt{\bar{\zeta}}}\right]$

where,

$B=\frac{a(x+1)(A+2 i S)}{8 \uparrow} \quad, \quad C=\frac{(x-1) A+2 i(x+1) S}{8 \uparrow}$

We see that, as pointed out also in $[3,4]$ for the case of simple biaxial tension, the stress and displacement functions [3.3] and [3.4] depend on the far field also through terms which are non singular at the crack tip. The arbitrary omission of ihese terms can lead to incorrect predictions on stress and displacement distributions and on crack propagation criteria.

4. THE ELASTIC STRAIN ENERGY DENSITY CRITERION

For the plane problem the elastic strain energy density at any point of the plane body is given by:

$$
W=\frac{1}{2}\left[\sigma_{x x} \frac{\partial u}{\partial x}+\sigma_{v y} \frac{\partial v}{\partial y}+\sigma_{x y}\left(\frac{\partial u}{\partial x}+\frac{\partial v}{\partial y}\right)\right]
$$


and in complex form:

$$
\begin{gathered}
W=\frac{1}{4}\left[\left(\sigma_{x x}+\sigma_{y y}\right)\left(\frac{\partial D}{\partial \zeta}+\frac{\partial D}{\partial \zeta}\right)+\left(\sigma_{x x}-\sigma_{y y}+2 i \sigma_{x y}\right)\right. \\
\left.\frac{\delta \bar{D}}{\delta \zeta}\left(+\sigma_{x x}-\sigma_{y y}-2 i \sigma_{x y}\right) \frac{\partial D}{\delta \bar{\zeta}}\right]
\end{gathered}
$$

After substitution of [3.3] and [3.4] into [4.2] we obtain the strain energy density near the crack tip which is:

$$
\begin{gathered}
W(\zeta \bar{\zeta})=\frac{1}{4}\left\{\frac{(x+1) A^{2}}{4 \mu}+\frac{1}{8 \mu \sqrt{\overline{\zeta \zeta}}}\left[(4 x-3)\left|S_{1}\right|^{2}+\left|S_{2}\right|^{2}\right]+\right. \\
+\frac{1}{4 \mu} \operatorname{Re}\left[2(x-1) \frac{S_{1}{ }^{2}}{\bar{\zeta}}-\frac{S_{1} S_{2}}{\bar{\zeta}} \sqrt{\frac{\bar{\zeta}}{\bar{\zeta}}}+A\left\{\frac{2 S_{1} \zeta}{\bar{\zeta} \sqrt{\bar{\zeta}}}+\right.\right. \\
\left.\left.\left.+\frac{4(x-1) S_{1}}{\sqrt{\bar{\zeta}}}-\frac{2 \bar{S}_{2}}{\sqrt{\bar{\zeta}}}\right)\right]\right\}
\end{gathered}
$$

According to Sih's theory (Sih, 1973) on the prediction of the direction of initial crack extension, crack growth will start along the radial direction for which the local elastic strain energy density attains a minimum. In view of this hypothesis the growing direction will be a solution of the following conditions:

$$
\left(\frac{\partial w}{\partial \varphi}\right)_{r_{0}}=0,\left(\frac{\partial w}{\partial \varphi^{2}}\right)_{r_{0}}>0
$$

where the radial distance $r_{0}$ is very small, but unspecified. 
Recently, an estimation of the range of $r_{\mathrm{o}}$ was given in the case of pure tension applied at infinity $[2,6]$. As shown in $[4,5]$ for the case of a biaxially loaded crack the value of $\varphi$ for which the elastic strain energy density attains a minimum is a function of $K$, as confirmed by experimental data. The same feature can be shown by substitution of [4.3] in [4.4]. Assuming that $S=n T$ where $n$ is a real positive number we can write the strain energy [4.3] as:

$$
\begin{gathered}
\frac{8 \mu}{T^{2}}\left(\frac{r_{0}}{a}\right) W(\varphi)=\frac{(K-1)^{2}(x+1)}{2}\left(\frac{r_{0}}{a}\right)+ \\
+\frac{(2 x-1)+n^{2}(2 x+3)}{4}+\frac{R e}{4}\left\{2(x-1)(1+i n)^{2} e^{i \varphi}-\right. \\
+(1+i n)(1+3 i n) e^{2 i \varphi}+2(K-1) \sqrt{\frac{2 r_{0}}{a}} \\
\left.\left[(2 x(1+i n)-(3-i n)) e^{i \varphi / 2}+(1+i n) e^{i^{5} / 2 \varphi}\right]\right\}
\end{gathered}
$$

Choosing $r_{0}=10^{-2} a$ in agreement with the condition on the distance $r_{o}$ from the crack border, the results of plotting the expression [4.5] vs. $\varphi$ in the case of plane stress are shown in figs. 3-6 with $K=-1,0,3,5,10$, the Poisson ratio $\nu=0.1,0.5$ and $n=0,1$. For $n=0$ we obtain the same results ans in $[2,3,4,5,5,6]$ corresponding to the biaxial tension-compression loads. The angle of initial crack extension corresponding to the minimum of the strain energy density occurs at $\varphi=0^{\circ}$ for $K=-1$ (and in general 
for $K \leq-1$ ) for all values of the Poisson ratio. For $K=0$ this angle occurs at $\varphi=0^{\circ}$ or $\varphi=90^{\circ}$ depending on the values of the Poisson ratio and for $K=3$ at $\varphi=90^{\circ}$. The graphs show also that if $K$ is very large $(K \geqslant 5)$ the angle of initial extension occurs at $\varphi=90^{\circ}$ for $\nu=0.1$ and an increase of the Poisson ratio shifts this angle at $\varphi=80^{\circ}$ (fig. 4). For $n=1$ the angle of initial crack extension is predicted to be greater than $90^{\circ}$ for any value of $K$ and $\nu$, that is the crack should bend back towards its centre. Since behaviour of this kind as never been reported this portion of the curves are shown by dashed lines. It seems that in the above circumstance the Sih criterion is inadequate.

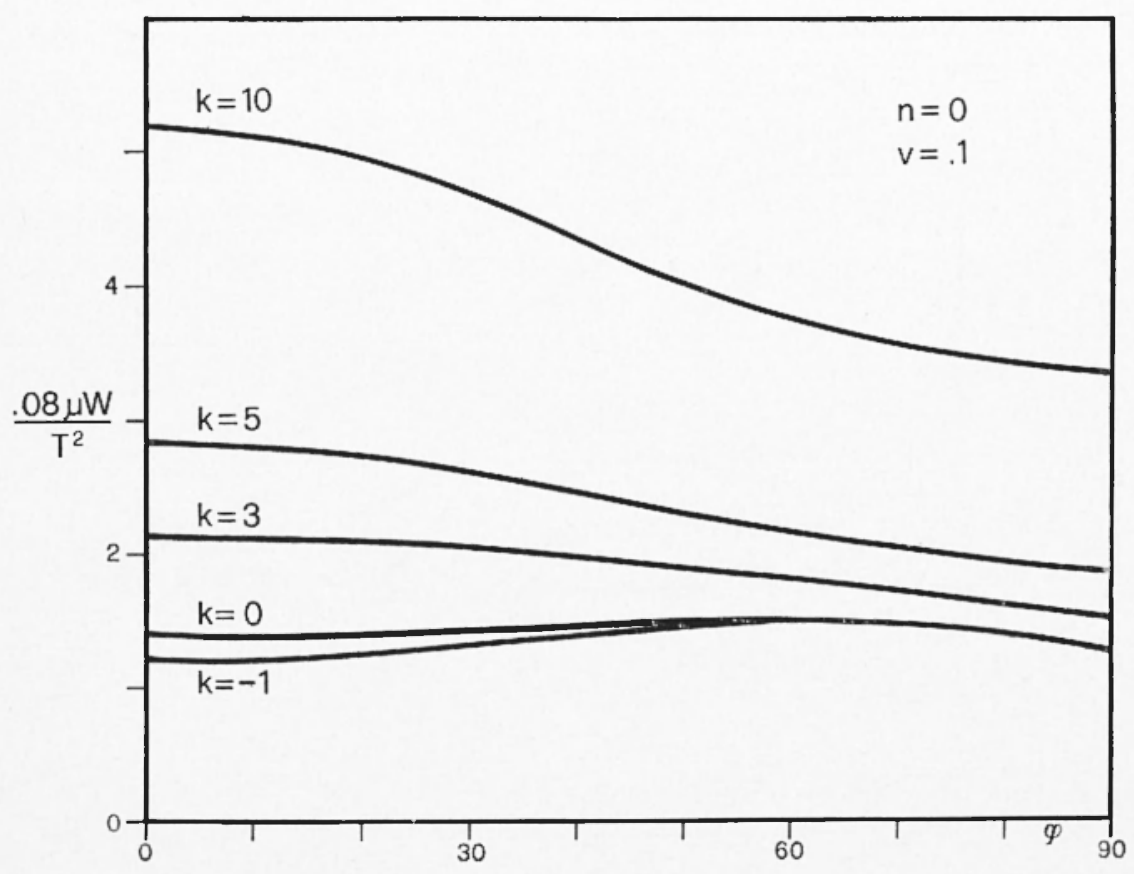

Fig. 3 
5. The MAXIMUM NORMAL STRESS CRITERION

The maximum normal stress criterion proposed firstly by Yoffe (1951) and reviewed by Erdogan and Sih (1963) is concerned with the direction of crack extension too and maintains that a crack will begin to extend radially along the plane on which the stress normal to this plane attains a positive maximum value. If $\varphi_{0}$ is the angle of the tangent to this plane, the criterion requires that, near the tip of the crack, we should have:

$$
\left(\sigma_{\varphi \varphi}\right)_{\rho_{\rho_{0}}}>0,\left(\frac{\partial \sigma_{\varphi \varphi}}{\partial \varphi}\right)_{\varphi_{0}}=0,\left(\frac{\delta \sigma_{\varphi \varphi}}{\partial \varphi^{2}}\right)_{\varphi_{\rho_{0}}}<0
$$

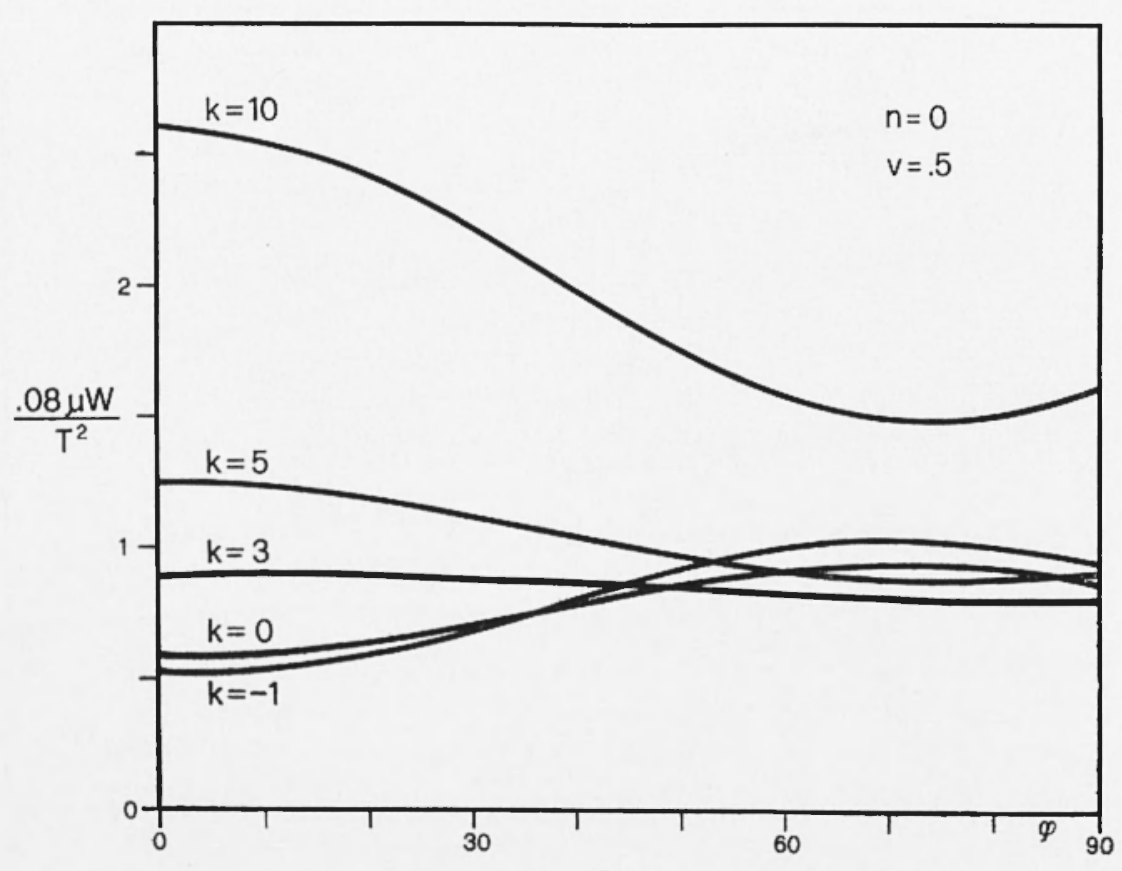

Fig. 4 
THE EFFECT OF THE STRESS FAR FIELD ECC.

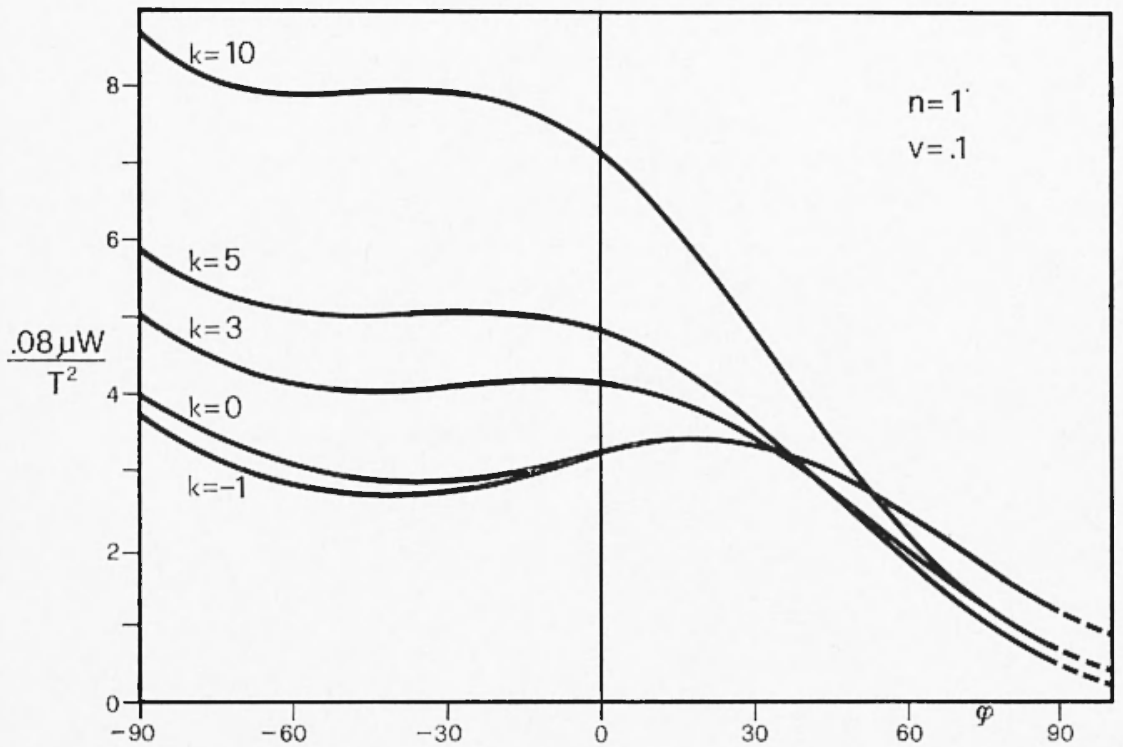

Fig. 5

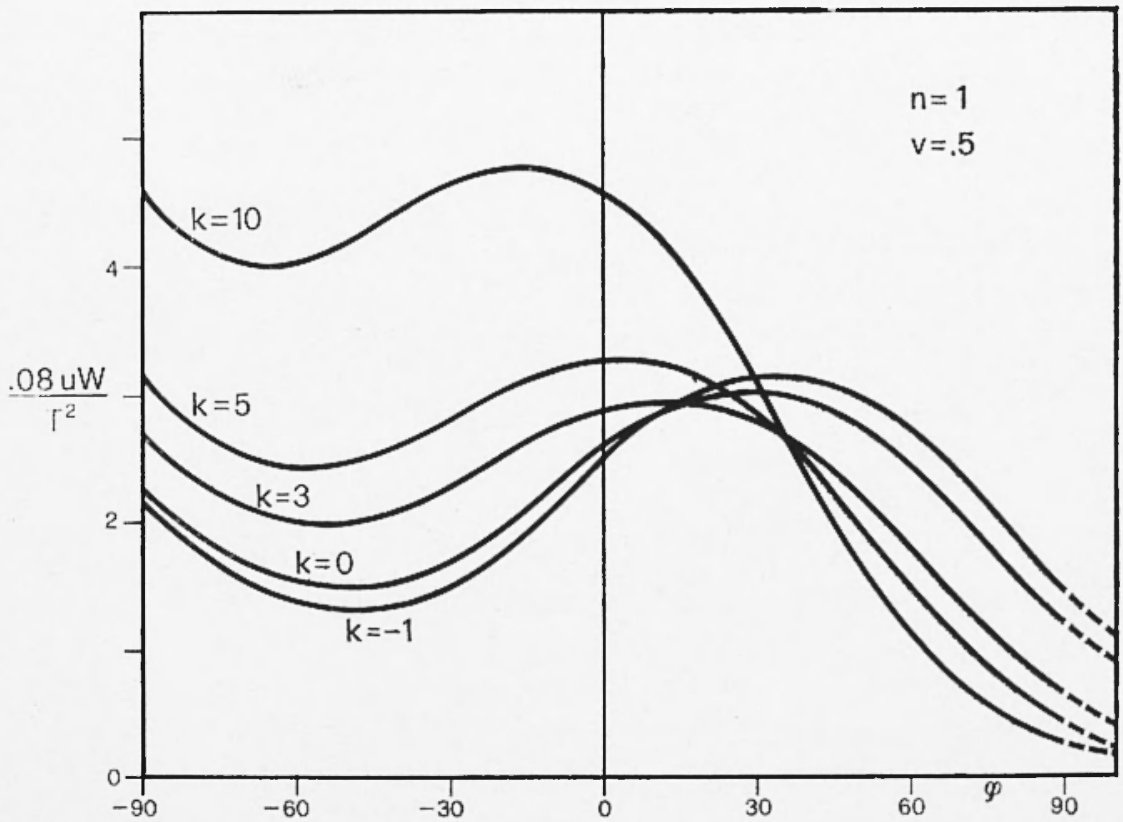

Fig. 6 


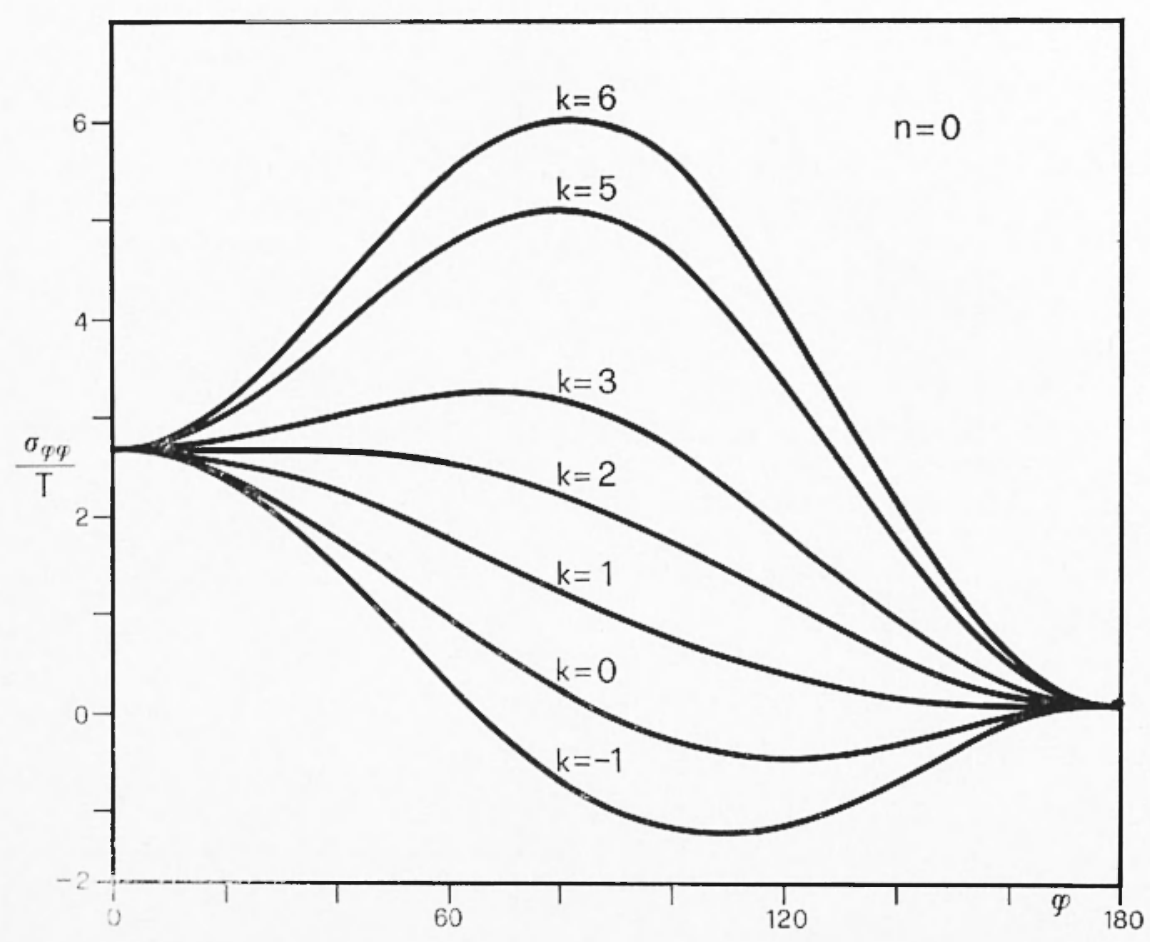

Fig. 7

Remembering that:

$$
\begin{gathered}
\sigma_{\varphi \varphi}=\frac{\sigma_{x x}+\sigma_{y y}}{2} ! \frac{\zeta}{\bar{\zeta}} \frac{\sigma_{y y}-\sigma_{x x}+2 i \sigma_{x y}}{4}+\frac{\bar{\zeta}}{\zeta} . \\
\frac{\sigma_{y y}-\sigma_{x x}-2 i \sigma_{x y}}{4}
\end{gathered}
$$

we obtain from [3.3] the following expression:

$$
\sigma_{\varphi \varphi}=\frac{A}{2}\left[1-\operatorname{Re}\left(\frac{\zeta}{\bar{\zeta}}\right)\right]+\frac{R e}{4}\left[\frac{3 S_{1}}{\sqrt{\zeta}}+\frac{S_{2} \sqrt{\zeta}}{\bar{\zeta}}\right]
$$


The variation of the angle $\varphi_{0}$ of initial crack extension is shown in figs. 7 and 8 where the function $\sigma_{G}^{-s} / T$ is plotted for different values of $K$. We have considered that $S=n T$ with $n=0,1$ and $r_{0} / a=0.07$. This last condition satisfies $[5.1]_{3}$ and the requirement tha $0<r / a \ll 1$. It. can be seen (fig. 7) that for $n=0$ and $K=2$ the maximum occurs at $\varphi_{0}=0^{\circ}$. As far as $K$ increases $\varphi_{0}$ increases up to $\varphi_{0} \simeq 80^{\circ}$ for $K=6$. In fig. 8 can be seen the influence of the shear stress field. We note that for $n=1$ the maximum of the normal stress occurs from $\varphi_{0}=-30^{\circ}$ to $\varphi_{0}=-70^{\circ}$ depending on the value of $K$.

Here again we find evidence of the influence of non singular terms contribution on the prediction of the angle of initial crack extension.

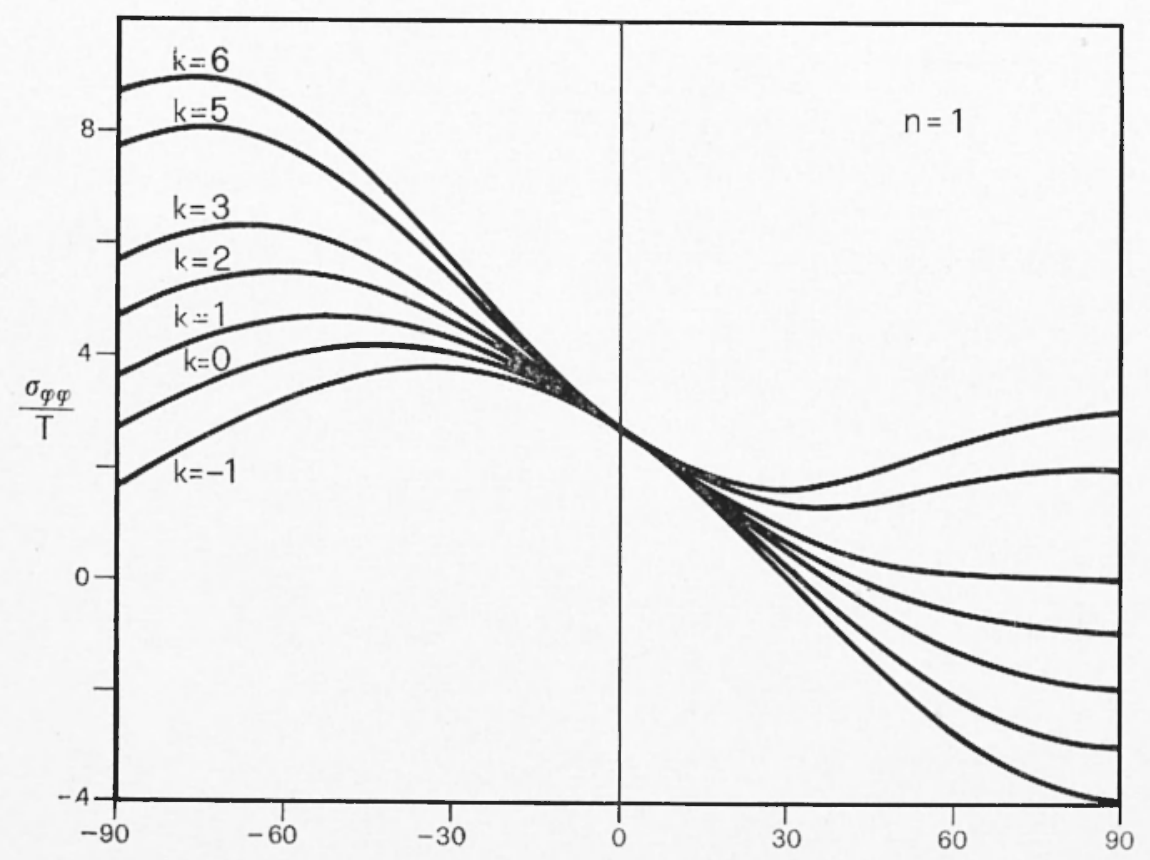

Fig. 8 
6. THE LOCAL ELASTIC STRAIN ENERGY RATE AND THE $J$-Vector

The elastic strain energy $W$ due to the presence of the crack is independent of the horizontal stress applied at infinity. In fact, we have:

$$
W=\frac{\pi a^{2}\left(T^{2}+S^{2}\right)(x+1)}{8 \mu}
$$

Moreover, the local elastic strain energy $W_{\mathrm{e}}$ obtained by integratio of [4.3] over a circular region centred at the crack tip with radius $0<r_{0} \ll 1$ has the following expression:

$$
\begin{aligned}
& W_{c}=\frac{\pi T^{2} r_{0}{ }^{2}}{8 \mu}\left[\frac{(2 x-1) T^{2}+(2 x+3)^{2}\left(S^{2}\right)}{2 T^{2}\left(r_{0}{ }^{2}\right)}\left\langle\frac{a_{i}}{r_{0}}\right\rangle+\right. \\
& \left.+\frac{16(K-1)(5 x-7)}{15 \pi \sqrt{2}} \sqrt{\frac{a}{r_{0}}}+\frac{(K-1)^{2}(x+1)}{2}\right]
\end{aligned}
$$

From [6.1] and [6.2] we obtain the global and local rates of change of the elastic strain energy with the crack size:

$$
\begin{gathered}
\frac{\partial W}{\partial a}=\frac{\pi(z+1)\left(T^{2}+S^{2}\right) a}{4 \mu} \\
\frac{\partial W}{\partial a}=\frac{\pi T^{2} r_{o}}{16 \mu}\left[\frac{(2 x-1) T^{2}+(2 x+3) S^{2}}{T^{2}}+\right. \\
\left.+\frac{16(K-1)(5 x-7)}{15 \pi \sqrt{2}} \sqrt{\frac{r_{0}}{a}}\right]
\end{gathered}
$$


We see that the global strain energy rate is independent of the biaxial stress at infinity which otherwise has a significant influence on the local elastic strain energy rate. This behaviour was pointed out also in $[4,5]$ for the case of simple biaxial tension at infinity.

Rice (1968) showed that the energy release rate for a twodimensional crack extending in its plane in a homogeneous material was equal to a path-independent integral $J_{1}$ formulated by Eshelby (1956) in the theory of lattice defects, and applied to crack problems by Sanders (1960) and Cherepanov (1969).

Knowles and Stenberg (1972) generalized $J_{I}$ to a vector $J_{k}$ corresponding to the energy release rate for a movement in any direction of the crack edge. Budiansky and Rice (1973) have shown how the formula may be simplified for a homogeneous isotropic material by using the theory of functions of a complex variable. Recently some authors (Bui, 1976); Carpinteri, 1978) showed that the energy release due to the extension of the crack along some direction is equal to the component of the $J$ vector along the direction of extension.

Here we formulate an expression for the $J$-integral in terms of the fundamental combinations of stresses and the derivatives of the complex displacement. The two components of the $J$ vector are:

$$
\begin{aligned}
& J_{1}=\oint W \mathrm{~d} y-\left(\sigma_{x x} \frac{\partial u}{\partial x}+\sigma_{x y} \frac{\partial v}{\partial x}\right) \mathrm{d} y+ \\
&+\left(\sigma_{x y} \frac{\partial u}{\partial x}+\sigma_{y y} \frac{\partial v}{\partial x}\right) \mathrm{d} x \\
& J_{2}=-\oint W \mathrm{~d} x+\left(\sigma_{x x} \frac{\delta u}{\partial y}+\sigma_{x y} \frac{\delta v}{\partial y}\right) \mathrm{d} y- \\
&-\left(\sigma_{x y} \frac{\partial u}{\partial y}+\sigma_{y y} \frac{\partial v}{\partial y}\right) \mathrm{d} x
\end{aligned}
$$


were $W$ is the strain energy density [4.3] and $\Gamma$ is a small circle surrounding the crack tip. Introducing the complex force acting on the element $d s$ of $\Gamma$ :

$$
\begin{gathered}
T \mathrm{~d} s=(T x+i T y) \mathrm{d} s=\left(\sigma_{x x} \mathrm{~d} y-\sigma_{x y} \mathrm{~d} x\right)+ \\
+i\left(\sigma_{x y} \mathrm{~d} y-\sigma_{y y} \mathrm{~d} x\right)
\end{gathered}
$$

we can write from [7.1] and [7.2]:

$$
J=J_{1}-i J_{2}=\oint_{\Gamma}\left[i w \mathrm{~d} \bar{\zeta}-\left(T \frac{\bar{\partial} \bar{D}}{\delta \zeta}+\bar{T} \frac{\partial D}{\delta \zeta}\right) \mathrm{d} s\right.
$$

This expression was obtained also by Hellen and Blackburn (1975). Moreover, we can write:

$$
J=i \underset{\Gamma}{\oint}\left[\left(T \frac{\partial \bar{D}}{\partial \zeta}+\bar{T} \frac{\partial D}{\partial \zeta}\right) \sqrt{\frac{\bar{\zeta}}{\zeta}}-W \frac{\bar{\zeta}}{\zeta}\right] \mathrm{d} \zeta
$$

together with:

$$
T=\frac{1}{2}\left[\sqrt{\frac{\bar{\zeta}}{\bar{\zeta}}}\left(\sigma_{x x}+\sigma_{y y}\right)+\sqrt{\frac{\bar{\zeta}}{\zeta}}\left(\sigma_{x x}-\sigma_{y v}+2 i \sigma_{x y}\right)\right]
$$


Finally, with the help of relations [4.2] and [7.6] the line integral [7.5] acquires the following form:

$$
\begin{gathered}
J=\frac{i}{4} \underset{\Gamma}{\oint}\left[\sigma_{x x}-\sigma_{y y}+2 i \sigma_{x y} \bar{\zeta} \frac{\partial \bar{D}}{\partial \zeta}+\sigma_{x x}-\sigma_{y y}-2 i \sigma_{x y}\right. \\
2 \zeta\left(\frac{\partial D}{\partial \zeta}-\zeta \frac{\partial D}{\partial \zeta}\right)+\left(\sigma_{x x}+\sigma_{y y}\right)\left(2 \zeta \frac{\partial \bar{D}}{\delta \zeta}+\right. \\
\left.\left.+\bar{\zeta} \frac{\partial D}{\delta \zeta}-\bar{\zeta} \frac{\partial \bar{D}}{\partial \zeta}\right)\right] \frac{\mathrm{d} \zeta}{\zeta}
\end{gathered}
$$

From [3.3] and [3.4] the integral [7.7] splits into three classes of integrals. The terms of the first class vanish identically; those of the second class, which are integrals containing the biaxial load parameter $K$ and the radius of $\mathrm{I}$, cancel one another out. The integrals of the third class are $K$ and path-independent. The resulting integral is:

$$
J=-\frac{i}{16 \mu} \oiint_{I^{\prime}}(x+1)\left(S_{1}{ }^{2}+\bar{S}_{1} S_{2}\right) \frac{\mathrm{d} \sigma}{\zeta}
$$

from which we obtain the well-known result:

$$
J=\frac{(x+1)(v+1)}{4 E}\left(K_{1}^{2}+K_{2}^{2}+2 i K_{1} K_{2}\right)
$$

Thus, the value of the $\bar{J}\left(J_{1}, J_{2}\right)$ is independent of the non singular terms appearing in the expressions of stresses and displacement, as was shown for $J_{1}$-integral by Eftis et al. (1977) in the case of a simple biaxial load. 


\section{CONCLUSION}

Assuming a two-dimensional crack as a model of a fault some results are obtained on the basis of recent progress in fracture mechanics which could be of interest in Geophysics.

As far as the crack tip behaviour is concerned the influence of the stress far field through terms which are non singular is pointed out. The arbitrary omission of these terms should cause an incorrect prediction on stress and displacement distributions and on local crack propagation criteria. It appears that the biaxiality of the stress far field affects only the local strain energy release rate and the independence of the $\bar{J}$-vector from the biaxial load parameter is shown. 
R E F E R E N C E S

Budiansky B, Rice J.R., 1973. - « J. Applied Mech. », 40, 201-205.

Bui H.D., 1976. - Arch. Mech. Stosowanij, 28, p. 649-659.

Carpinteri A., 1978. - Tech. Note. "Ist. di Scienza delle Costruzioni ", Univ. di Bologna.

Carpinteri A., Di Tommaso A., Viola E., 1978. - Proceedings 40 Congresso Naz. AIMETA, Firenze.

Cherepanov G.L., 1967. - P.M.M., 25, p. 476-488.

Di Tommaso A., Nobile L., Viola E., 1976. - Proceedings 30 Congresso Naz. AIMETA, Firenze, 1976.

Eftis J., Subramonian N., Liebowitz H., 1977. - Eng. Fract. Mech., p. 182-210.

Eftis J., Subramonian N., Liebowitz H. ,1977. - Eng. Fract. Mech., p. 753-764.

ERdogan F., Sin G.C., 1963. - J. Bas. Eng., 85D, p. 519-525.

Eshelby J.R., 1956. - Solid State Phisics, 3.

Hellen T.K., Blackburn W.S., 1975. - Int. J. of Fract., 11, p. 605-617.

Inglis C.E., 1913. - "Trans. Inst. Naval Arch. », 55, Part 1, p. 219-230.

Knowles J.K., Stenderg E., 1972. - Arch. Rat. Mech., 44, p. 187-211.

Muskelishvili N.I., 1953. - Some Basic Problems of the Mathematical Theory of Elasticity. Groniningen, Noordhoff.

Rice J.R., 1968. - «J. Applied Mech. », 35, p. 379-386.

Sanders J.L., 1960. - J. Applied Mech., 27, p. 352-353.

SIH G.C., 1973. - Methods of analysis and solution of crack problems. G.C. Sih, Noordhoff Int.

Viola E., 1978. - Tech. Note, Ist. di Scienza delle Costruzioni, Univ. di Bologna.

Yoffe E.H., 1951. - Phyl. Magazine, 32, p. 739-750. 